\title{
Validation of the novel susceptibility loci for prostate cancer in a Chinese population
}

\author{
YISHUO WU ${ }^{1,2^{*}}$, HAITAO CHEN ${ }^{3 *}$, YING JI $^{1 *}$, RONG NA $^{1,2}$, ZENGNAN MO $^{4}$, DINGWEI YE ${ }^{5}$, \\ MEILIN WANG ${ }^{6}$, JUN QI ${ }^{7}$, XIAOLING LIN ${ }^{2,3}$, QIANG DING ${ }^{1,2}$, JIANFENG XU ${ }^{2,8}$, \\ S. LILLY ZHENG ${ }^{8}$, YINGHAO SUN ${ }^{9}$ and WEI MENG ${ }^{10}$ \\ ${ }^{1}$ Department of Urology, Huashan Hospital, Fudan University; ${ }^{2}$ Urology Research Center, Fudan University; \\ ${ }^{3}$ Center for Genomic Translational Medicine and Prevention, School of Public Health, Fudan University, Shanghai 200000; \\ ${ }^{4}$ Center for Genomic and Personalized Medicine, Guangxi Medical University, Nanning, Guangxi 530000; \\ ${ }^{5}$ Department of Urology, Shanghai Cancer Center, Fudan University, Shanghai 200000; ${ }^{6}$ Department of Molecular and \\ Genetic Toxicology, The Key Laboratory of Modern Toxicology of The Ministry of Education, School of Public Health, \\ Nanjing Medical University, Nanjing, Jiangsu 210000; ${ }^{7}$ Department of Urology, Xinhua Hospital, \\ Shanghai Jiaotong University School of Medicine, Shanghai 200000, P.R. China; ${ }^{8}$ Program for Personalized Cancer Care, \\ NorthShore University Health System, Evanston, IL 60201, USA; ${ }^{9}$ Department of Urology, Changhai Hospital, \\ The Second Military Medical University; ${ }^{10}$ Department of Epidemiology, School of Public Health, \\ Fudan University, Shanghai 200000, P.R. China
}

Received January 14,2017; Accepted October 24, 2017

DOI: $10.3892 / 01.2017 .7602$

\begin{abstract}
The present study evaluated 23 newly identified susceptibility loci for prostate cancer (PCa) in a Chinese population and assessed whether any validated loci were associated with the genetic risk score (GRS) of PCa in a Chinese population. A total of 1,417 patients with PCa and 1,008 controls were recruited in the present study. The association of each single nucleotide polymorphism (SNP) with PCa risk and PCa aggressiveness was analyzed. The predictive ability of two GRSs based on 30 SNPs (GRS30) and the 9 most significant SNPs (GRS9) in the Chinese population were also compared. Among the 19 SNPs evaluated, 1 SNP (rs7153648 at 14q23) was associated with $\mathrm{PCa}$ risk [odds ratio $(\mathrm{OR})=1.206, \mathrm{P}<0.05)$ ] and 1 SNP (rs636291 at 1p23) was associated with PCa aggressiveness $(\mathrm{OR}=1.123, \mathrm{P}<0.05)$. GRS30 and GRS9 were significantly increased in patients with PCa compared with that among non-PCa controls. The areas under receiver operating
\end{abstract}

Correspondence to: Dr Qiang Ding, Department of Urology, Huashan Hospital, Fudan University, 12 Mid-Wulumuqi Road, Shanghai 200000, P.R. China

E-mail: qiangd_urology@126.com

Professor Jianfeng Xu, Urology Research Center, Fudan University, 12 Mid-Wulumuqi Road, Shanghai 200000, P.R. China

E-mail: jxu8088@gmail.com

*Contributed equally

Key words: prostate cancer, single nucleotide polymorphisms, genetic risk score, Chinese characteristic curves of GRS 9 and GRS 30 were similar $(0.792$ for GRS9 vs. 0.7994 for GRS30, P=0.138). To conclude, among the 19 SNPs evaluated, only 1 SNP was associated with PCa risk in the Chinese population. SNPs that were weakly associated with $\mathrm{PCa}$ were unlikely to improve the predictive ability of existing GRS in the Chinese population.

\section{Introduction}

Prostate cancer (PCa) is the second most common cancer and one of the leading causes of mortality among males worldwide by 2012 (1). The incidence of PCa in China is considered reduced compared with that in Western countries; however, it has been progressively increasing over the past 30 years (2).

Genetic susceptibility to PCa has been well established and almost 100 common risk loci have been identified by genome wide association studies (GWAS) among European, African-American, Japanese and Chinese populations $(3,4)$. However, only 10 of these loci were initially identified from GWAS in Japanese and Chinese populations. Among previous evaluation and validation studies, a part of the loci was revealed to be associated with PCa risk in Chinese population (5). Since these risk-associated single nucleotide polymorphisms (SNPs) exhibited a cumulative effect on PCa risk, the genetic risk scores (GRS) derived from PCa risk-associated SNPs were able to evaluate an individual's risk of PCa. The GRS based on the Chinese population is established and demonstrated to be a significant predictor of biopsy outcome in previous studies (5-9).

With an increasing sample size used in GWAS through combined data, a meta-analysis of a multi-ethnic population, which included 87,040 individuals, identified 23 new susceptibility loci for PCa (including 15 in European, 7 in 
multiethnic and 1 in the early onset analysis) (10). These PCa risk-associated alleles exhibited decreased effects with odds ratios (ORs) ranging between 1.06 and 1.14 (10). However, since the Chinese population was not included in the study, the effects of these 23 novel risk variants in individuals of Chinese descent remains unknown.

The objective of the present study was to evaluate the 23 newly identified susceptibility loci for PCa in a Chinese population and assess whether any validated loci contributed to the GRS in predicting the risk of PCa in a Chinese population.

\section{Materials and methods}

Population. The baseline characteristics of the present study subjects were summarized (Table I). A total of 2,425 subjects including 1,417 patients with PCa and 1,008 controls were recruited in the present study. All patients were part of the China PCa consortium from the southeast of China (11-13) recruited during January 2010 and December 2011, from which data were obtained. All cases were pathologically diagnosed with primary PCa and all the controls were recruited from the community or selected from subjects who had undergone routine physical examination in local hospitals. Written informed consent was obtained from subjects for their participation in the present study and a blood sample was taken from each subject at the time of recruitment for DNA extraction. The present study was reviewed and approved by the Institutional Review Board of every participating institution.

Genotyping and quality control. DNA samples were genotyped in the Center for Cancer Genomics at Wake Forest University (Winston-Salem, NC, USA) using the Illumina HumanOmniExpress BeadChips (Illumina, Inc., San Diego, CA, USA), which included 731,458 SNPs. For PCa risk-associated SNPs that were not included in the GWAS array, imputation was performed using IMPUTE 2.2.2 based on the combined data of the 1,000 Genomes project and HapMap3 data (14). A posterior probability of $>0.9$ was applied to call imputed genotypes. Imputed SNPs were excluded if they exhibited: i) A call rate $<95 \%$; ii) a minor allele frequency $<0.05$; or iii) $\mathrm{P}<1 \times 10^{-3}$ in a Hardy-Weinberg equilibrium test in controls, as previously described (13).

Assessment of genetic risk. A GRS was calculated for each subject based on genotypes of the SNPs and weighted by their ORs and risk allele frequency, as described previously (15). GRS was calculated as

$$
\mathrm{GRS}=\prod_{i=1}^{n} \frac{O R_{i}^{g_{i}}}{W_{i}},
$$

where $g_{\mathrm{i}}$ is the genotype of SNP $i$ for an individual (0, homozygous of non-risk allele; 1 , heterozygous; 2 homozygous of risk allele). $O R_{\mathrm{i}}$ is the OR of SNP $i$ estimated from external study (16), $W_{\mathrm{i}}$ is the average population risk of SNP $i$, calculated as $W_{\mathrm{i}}=f_{\mathrm{i}}^{2} O R_{\mathrm{i}}^{2}+2 f_{\mathrm{i}}\left(1-f_{\mathrm{i}}\right) O R i+\left(1-f_{\mathrm{i}}\right)^{2}$, where $f_{\mathrm{i}}$ is the risk allele frequency of SNP $i$ based on the 1,000 Genome Project of the CHB (Han Chinese in Bejing, China) population (17). Therefore, a GRS value of 1.0 represents a population average risk.
Table I. Characteristics of study population.

\begin{tabular}{lcc}
\hline Variables & PCa cases $(\mathrm{n}=1,417)$ & Controls $(\mathrm{n}=1,008)$ \\
\hline Age, years ${ }^{\mathrm{a}, \mathrm{b}}$ & $71.3 \pm 8.1$ & $62.1 \pm 10.0$ \\
PSA, ng/ml $\mathrm{ml}^{\mathrm{a}, \mathrm{c}}$ & & \\
$0-3.99$ & $54(4.0)$ & $965(95.9)$ \\
$4-9.99$ & $187(14.0)$ & $32(3.2)$ \\
$10-19.99$ & $305(22.8)$ & $6(0.6)$ \\
$\geq 20$ & $791(59.2)$ & $3(0.3)$ \\
Missing & $80(5.6)$ & $2(0.2)$ \\
Gleason score & & \\
$\leq 7$ & $809(60.1)$ & N/A \\
$\geq 8$ & $537(39.9)$ & N/A \\
Missing & $71(5)$ & N/A \\
\hline
\end{tabular}

${ }^{\mathrm{a}} \mathrm{At}$ the time of diagnosis for cases or at recruitment for controls. ${ }^{\mathrm{b}} \mathrm{Data}$ are presented as the mean \pm standard deviation. ${ }^{\mathrm{C}}$ Data are presented as $\mathrm{n}(\%)$. PSA, prostate-specific antigen; N/A, not applicable; $\mathrm{PCa}$, prostate cancer.

Statistical analysis. A logistic regression model was used to analyze the association of each SNP with PCa risk, assuming an additive genetic model, which was implemented in PLINK version 1.07 (18). ORs and 95\% confident intervals (CIs) were estimated from logistic regression analysis with adjustment for age and the highest eigen value. Student's t-tests were used to analyze the differences in means of normally distributed variables between 2 groups. For variables that were not normally distributed, 2 tests were performed: i) A nonparametric method using the Wilcoxon rank sum test and ii) Student's t-tests for different means between 2 groups following log-transformation. Differences in binary variables were investigated using $\chi^{2}$ tests. Area under the receiver operating characteristic curve (AUC) was used to evaluate the performance of GRS in discriminating between 2 groups of subjects. The difference between two AUCs was determined using Delong's test (19). $\mathrm{P}<0.05$ was considered to indicate a statistically significant difference. All statistical analyses were performed using SPSS 19.0 (SPSS; IBM Corporation, Armonk, NY, USA).

\section{Results}

SNPs and PCa risk. The present study evaluated 19 newly reported SNPs. Among the 19 SNPs, only 1 (rs7153648 at 14q23) was associated with PCa risk in the China PCa cohort $(\mathrm{OR}=1.206, \mathrm{P}<0.05)$. The direction of the effect was consistent with the previous multiethnic meta-analysis (10). The other 18 SNPs that had previously demonstrated genome-wide significance in European ancestry meta-analysis and multiethnic meta-analysis (Table II) either were not associated with PCa risk or did not demonstrate the same magnitude of effect in the Chinese population investigated in the present study.

SNPs and PCa aggressiveness. The association between the 19 SNPs and PCa aggressiveness was also investigated (cases with a Gleason score $\geq 7$; Table III). The results did not demonstrate 


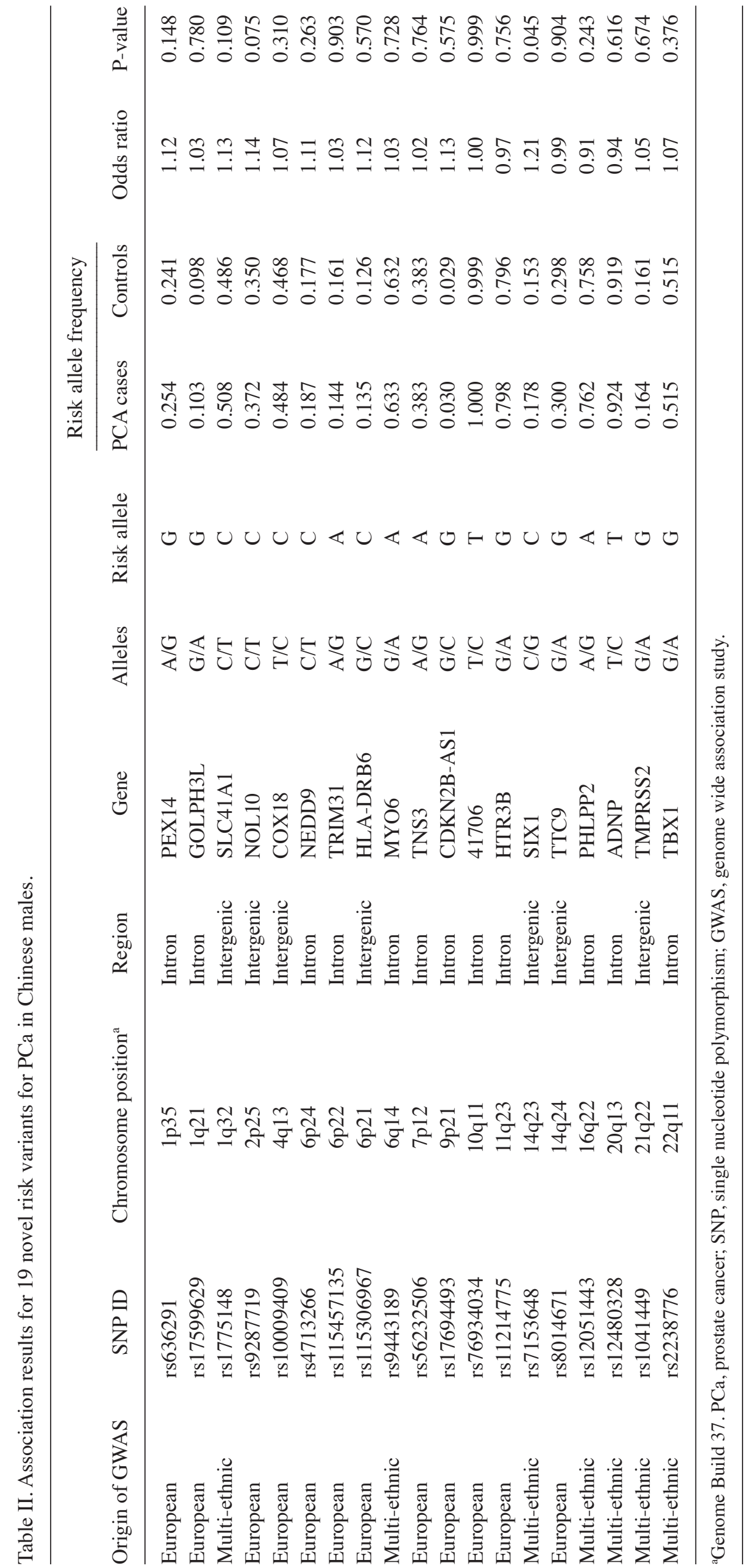




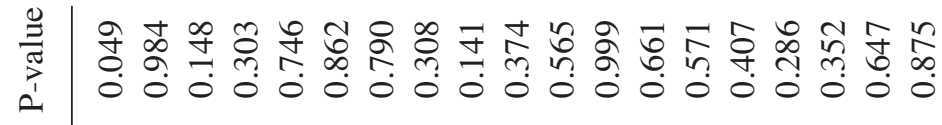

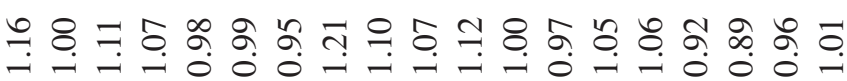

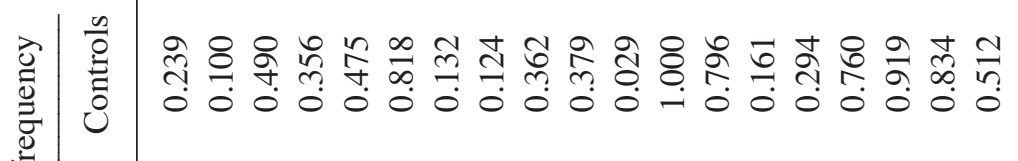

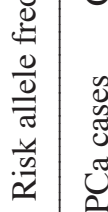

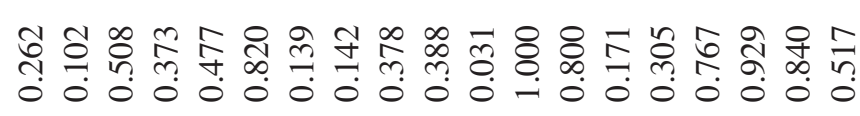
$\frac{\frac{0}{0}}{\frac{0}{\pi}}$

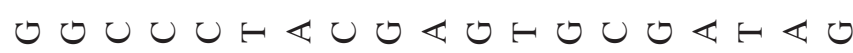

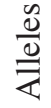

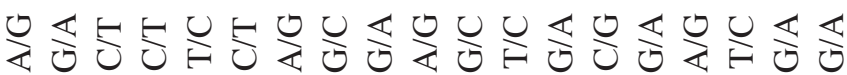

ڤั๊

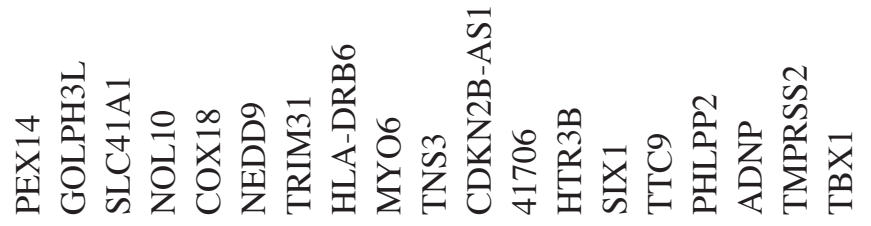

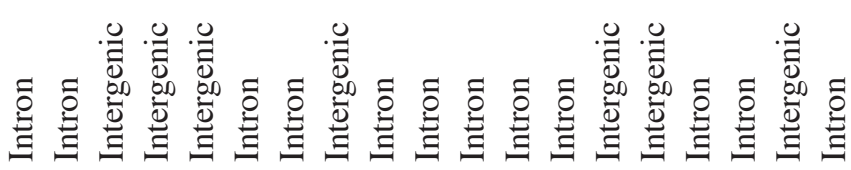

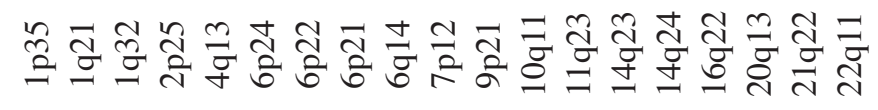

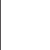

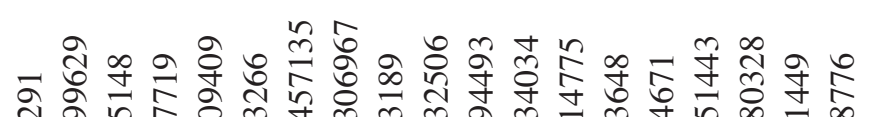

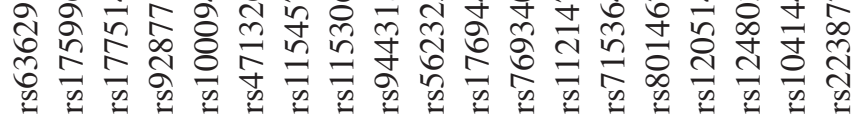


Table IV. Genetic score and prostate biopsy outcomes.

\begin{tabular}{lcc}
\hline Parameter & 9 SNPs & 30 SNPs \\
\hline Genetic score $^{\mathrm{a}}$ & & \\
$\mathrm{PCa}$ & $1.26 \pm 0.72$ & $1.44 \pm 1.18$ \\
Non-PCa & $0.99 \pm 0.53$ & $0.96 \pm 0.73$ \\
P-value & $3.71 \times 10^{-28}$ & $7.44 \times 10^{-41}$ \\
Association with $\mathrm{PCa}^{\mathrm{b}}$ & & 1 \\
Genetic score $\leq 1.0$ & 1 & $2.25(1.96-2.58)$ \\
Genetic score $>1.0$ & $2.47(2.05-2.97)$ & 0.799 \\
P-value & $6.90 \times 10^{-22}$ & \\
Discrimination of PCa & & \\
AUC & 0.792 & \\
P-value & & 0.138 \\
(AUC comparison) & & \\
\end{tabular}

${ }^{a}$ Data presented as the mean \pm standard deviation. ${ }^{b}$ Data presented as odds ratio (95\% confidence interval). PCa, prostate cancer; AUC, areas under receiver operating characteristic curves; SNP, single nucleotide polymorphism.

a significant association between rs7153648 and PCa aggressiveness, whereas rs636291 at 1p23 was significantly associated with $\mathrm{PCa}$ aggressiveness $(\mathrm{OR}=1.123, \mathrm{P}<0.05)$.

SNPs, GRS and PCa. GRS was calculated using rs7153648 and 29 previously implicated SNPs (10). The mean GRS based on the 30 SNPs (GRS30) was significantly increased in patients with $\mathrm{PCa}$ compared with that among non-PCa individuals ( 1.439 vs. $0.961, \mathrm{P}=7.44 \times 10^{-41}$; Table IV). As reported in a previous study, it would be more efficient and reliable to calculate GRS using race-specific disease-associated SNPs that demonstrated genome-wide significance (20). Therefore, in the present study, GRS was also calculated based on the 9 strongest SNPs previously reported in individuals of Asian descent (GRS9; Table V) (16). The mean GRS based on 9 SNPs was 1.26 in patients with $\mathrm{PCa}$ and 0.99 in non-PCa controls $\left(\mathrm{P}=3.71 \times 10^{-28}\right)$.

Following adjustment for age (Table IV), GRS9 and GRS30 remained significantly associated with $\mathrm{PCa}$ (all $\mathrm{P}<0.01$ ). The OR of the GRS30 for the prediction of PCa risk was 2.25 (95\% CI, 1.976-2.598; $\left.\mathrm{P}=2.97 \times 10^{-31}\right)$, decreased compared with that of GRS9 (OR=2.468; 95\% CI, 2.053-2.967; $\mathrm{P}=6.9 \times 10^{-22}$ ), although no significant differences were identified. When comparing the predictive ability of the GRS9 and GRS30, the AUCs were similar (0.792 for GRS9 vs. 0.7994 for GRS30, $\mathrm{P}=0.138$ ).

\section{Discussion}

Genetic susceptibility is a major risk factor for $\mathrm{PCa}$ and is estimated to account for $42 \%$ of variation in the disease (21). In the past few years, GWAS and meta-analysis of combined data have identified 99 genomic variants associated with PCa in multiple populations of European, African-American, Japanese, Latino and Chinese ancestry (10). In the present study, 23 novel susceptibility loci detected in European

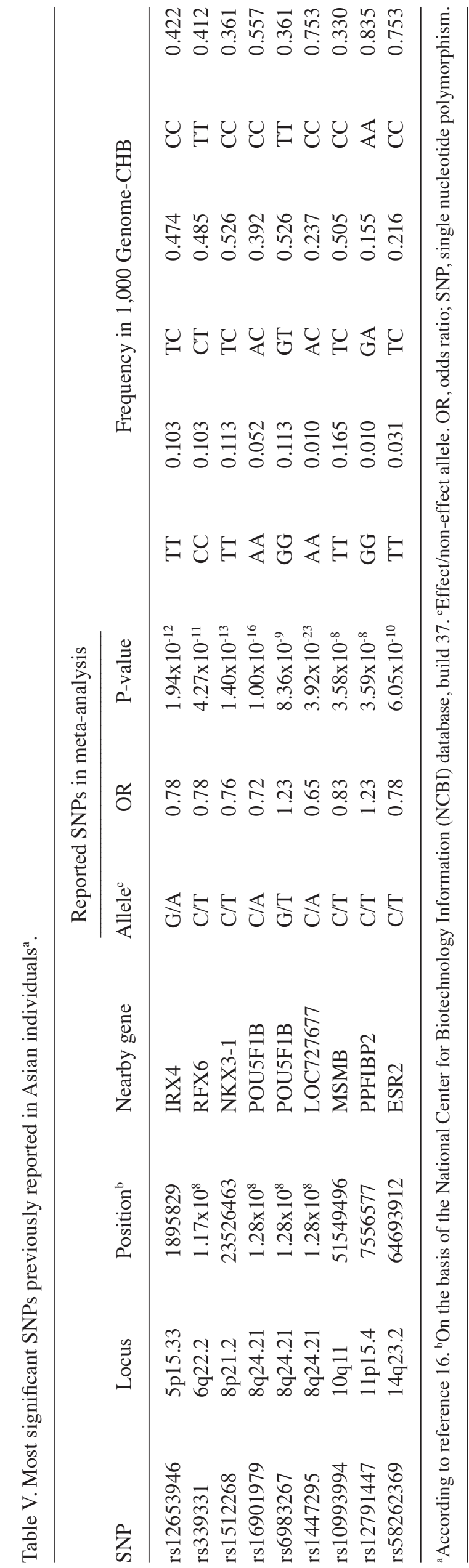




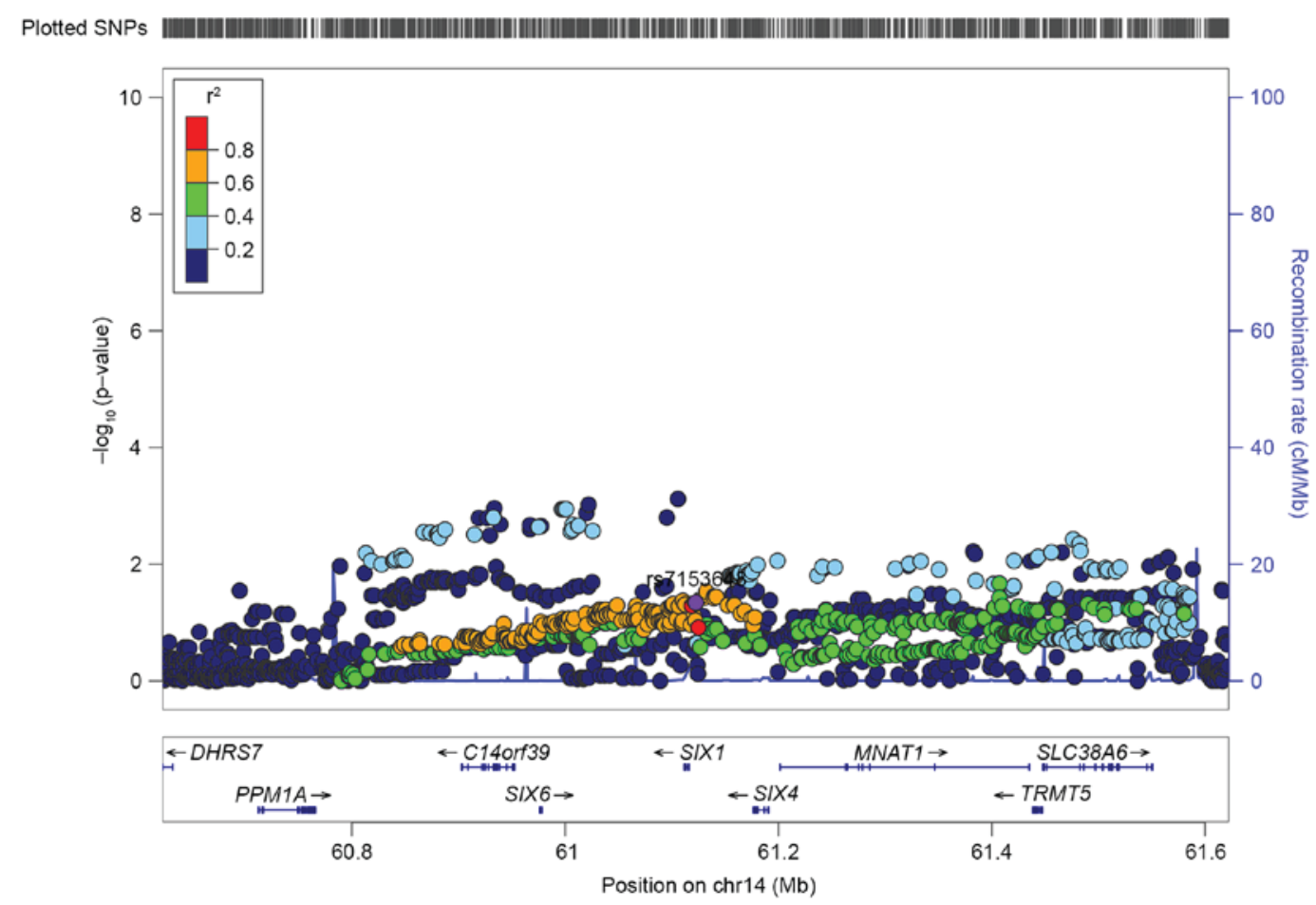

Figure 1. Regional information of rs7153648 at 14q23 (build: hg19). SNP, single nucleotide polymorphism.

ancestry or multi-ethnic analysis were investigated and their association in a Chinese population was evaluated.

Of the 19 SNPs evaluated in the present study, only 1 was identified to be associated with PCa. The estimate of risk of this SNP in the Chinese population was similar to that in European and multi-ethnic populations (10). Despite reaching genome-wide significance in European or multi-ethnic populations, the other 18 loci were not identified to be significant in the population of the present study. The discrepancy may be explained in multiple ways. First, since the effects of 18 SNPs (not including rs636291 at 1p36) were relatively low, with ORs ranging between 1.06 and 1.13 , the present study may not possess the power to identify the small effects of these SNPs. This was also one of the reasons why rs7153648 did not reach a significant level following Bonferroni correction $(\mathrm{P}=0.05 / 19)$. Second, the risk allele frequencies in European and Chinese ancestry differed between SNPs evaluated (Table II); this difference may also influence the detection of significant effects of these SNPs in populations of Chinese ancestry. Finally, besides the different genetic backgrounds between European ancestry (or other populations) and Chinese ancestry, environmental factors, dietary-habit and other non-genetic factors may also affect the penetrance of these alleles, which may result in the difference of risk profiles.

When evaluating the association between the 19 SNPs and aggressive $\mathrm{PCa}$ (Gleason score, $\geq 7$ ), the results demonstrated that rs636291 at $1 \mathrm{p} 36$ reached a significant level $(\mathrm{P}<0.05)$. This SNP reached genome-wide significance in early onset disease in European ancestry (10); however, a similar analysis could not be performed in the present study due to the lack of cases (only 34 patients with PCa were diagnosed $<55$ years of age). Nevertheless, this result may indicate that this risk variant was associated with more advanced $\mathrm{PCa}$ and should be further validated in an independent study.
In the comparison of the two GRS-based risk models, the results revealed that the performance was approximately the same between the two models. This may be attributed to the fact that certain risk variants were not strongly associated with $\mathrm{PCa}$ and others conferred a decreased effect to the risk of PCa in Chinese population compared with that in European whites. In a previous study, the plateau effect of PCa risk-associated SNPs was evaluated in predicting PCa in a Chinese population and it was identified that the predictive performance increased when the top 13 highest impact PCa risk-associated SNPs were included in the GRS (9). The results were similar in the present study; therefore, this may indicate that further SNPs weakly associated with $\mathrm{PCa}$ may not improve the predictive performance of GRS for PCa. Therefore, GRS only including the strongest SNPs may be appropriate while balancing the predictive performance and economic benefit.

In the present study, the variant rs7153648 at 14q23 that we demonstrated to be associated with PCa is located in the intergenic region of SIX homeobox 1. The regional information of the confirmed SNP (rs7153648) was presented (Fig. 1). In the LocusZoom plots of this loci, multiple SNPs located upstream of rs7153648 demonstrated marked association $(\mathrm{P}<0.01)$ but a weak correlation (dark blue circles), which may suggest the presence of multiple potential independent association signals. Variant rs636291 at 1p36, which was associated with early-onset $\mathrm{PCa}$ in European ancestry and was identified to be associated with aggressive $\mathrm{PCa}$ in Chinese ancestry in the current study, is located in intron 2 of peroxisomal biogenesis factor 14 and is associated with a variant (rs616488) reported in a GWAS of breast cancer (22).

There were multiple limitations to the present study. First, only 19 SNPs, rather than 23 of the novel identified loci, were genotyped or imputed due to 4 SNPs not being included in the GWAS panel and failing to impute using the CHB population 
of the 1,000 Genome project. Among the 4 SNPs, rs80130819 at $12 \mathrm{q} 13$ was not polymorphous in the $\mathrm{CHB}$ population, while the remaining 3 were polymorphous in the $\mathrm{CHB}$ population. Second, due to the open nature of the China PCa cohort, the clinical characterization of the cases was not consistent between distinct hospitals (e.g., Gleason score diagnosis in the present study), which limited further analysis of clinical phenotypes.

To conclude, by evaluating 19 PCa risk-associated SNPs identified in a large meta-analysis of GWAS from a European and multiethnic population, the results of the present study identified 1 SNP that was associated with PCa risk and 1 that was associated with aggressive $\mathrm{PCa}$ in a Chinese population. However, the validated small-effect SNP and other SNPs that weakly associated with PCa are not likely to improve the predictive ability of existing GRS in Chinese populations.

\section{Acknowledgements}

The present study was supported by the National Natural Science Foundation of China (grant nos. 81202269 and 81402339), the Clinical Science and Technology Innovation Project of Shanghai Shen Kang Hospital Development Center (grant no. SHDC12015105) and Scientific Research Project supported by Huashan Hospital, Fudan University (grant no. 2016QD079).

\section{References}

1. Torre LA, Bray F, Siegel RL, Ferlay J, Lortet-Tieulent J and Jemal A: Global cancer statistics, 2012. CA Cancer J Clin 65: 87-108, 2015.

2. Liu M, Wang JY, Zhang YG, Zhu SC, Lu ZH and Wan B: Detection of urological and male genital tumors diagnosed in Beijing Hospital 1995-2004. Zhonghua Yi Xue Za Zhi 87: 2423-2425, 2007 (In Chinese).

3. Xu J, Sun J and Zheng SL: Prostate cancer risk-associated genetic markers and their potential clinical utility. Asian J Androl 15 314-322, 2013

4. Eeles R, Goh C, Castro E, Bancroft E, Guy M, Al Olama AA, Easton D and Kote-Jarai Z: The genetic epidemiology of prostate cancer and its clinical implications. Nat Rev Urol 11: 18-31, 2014

5. Na R, Liu F, Zhang P, Ye D, Xu C, Shao Q, Qi J, Wang X, Chen Z, Wang M, et al: Evaluation of reported prostate cancer risk-associated SNPs from genome-wide association studies of various racial populations in Chinese men. Prostate 73: 1623-1635, 2013.

6. Aly M, Wiklund F, Xu J, Isaacs WB, Eklund M, D'Amato M, Adolfsson J and Grönberg H: Polygenic risk score improves prostate cancer risk prediction: Results from the Stockholm-1 cohort study. Eur Urol 60: 21-28, 2011.

7. Kader AK, Sun J, Reck BH, Newcombe PJ, Kim ST, Hsu FC, D'Agostino RB Jr, Tao S, Zhang Z, Turner AR, et al: Potential impact of adding genetic markers to clinical parameters in predicting prostate biopsy outcomes in men following an initial negative biopsy: Findings from the REDUCE trial. Eur Urol 62: 953-961, 2012.

8. Jiang H, Liu F, Wang Z, Na R, Zhang L, Wu Y, Zheng J, Lin X, Jiang D, Sun J, et al: Prediction of prostate cancer from prostate biopsy in Chinese men using a genetic score derived from 24 prostate cancer risk-associated SNPs. Prostate 73: 1651-1659, 2013.
9. Ren S, Xu J, Zhou T, Jiang H, Chen H, Liu F, Na R, Zhang L, Wu Y, Sun J, et al: Plateau effect of prostate cancer risk-associated SNPs in discriminating prostate biopsy outcomes. Prostate 73: 1824-1835, 2013.

10. Al Olama AA, Kote-Jarai Z, Berndt SI, Conti DV, Schumacher F, Han Y, Benlloch S, Hazelett DJ, Wang Z, Saunders E, et al: A meta-analysis of 87,040 individuals identifies 23 new susceptibility loci for prostate cancer. Nature Genetics 46: 1103-1109, 2014.

11. Liu F, Hsing AW, Wang X, Shao Q, Qi J, Ye Y, Wang Z, Chen H, Gao X, Wang G, et al: Systematic confirmation study of reported prostate cancer risk-associated single nucleotide polymorphisms in Chinese men. Cancer Sci 102: 1916-1920, 2011.

12. Wang M, Liu F, Hsing AW, Wang X, Shao Q, Qi J, Ye Y, Wang Z, Chen H, Gao X, et al: Replication and cumulative effects of GWAS-identified genetic variations for prostate cancer in Asians: A case-control study in the ChinaPCa consortium. Carcinogenesis 33: 356-360, 2012.

13. Xu J, Mo Z, Ye D, Wang M, Liu F, Jin G, Xu C, Wang X, Shao Q, Chen Z, et al: Genome-wide association study in Chinese men identifies two new prostate cancer risk loci at $9 \mathrm{q} 31.2$ and $19 \mathrm{q} 13.4$. Nat Genet 44: 1231-1235, 2012.

14. Marchini J, Howie B, Myers S, McVean G and Donnelly P: A new multipoint method for genome-wide association studies by imputation of genotypes. Nat Genet 39: 906-913, 2007.

15. Wu Y, Zhang N, Li K, Chen H, Lin X, Yu Y, Gou Y, Hou J, Jiang D, Na R, et al: Genetic scores based on risk-associated single nucleotide polymorphisms (SNPs) can reveal inherited risk of renal cell carcinoma. Oncotarget 7: 18631-18637, 2016.

16. Wang M, Takahashi A, Liu F, Ye D, Ding Q, Qin C, Yin C, Zhang Z, Matsuda K, Kubo M, et al: Large-scale association analysis in Asians identifies new susceptibility loci for prostate cancer. Nat Commun 6: 8469, 2015.

17. 1000 Genomes Project Consortium, Auton A, Brooks LD, Durbin RM, Garrison EP, Kang HM, Korbel JO, Marchini JL, McCarthy S, McVean GA and Abecasis GR: A global reference for human genetic variation. Nature 526: 68-74, 2015.

18. Purcell S, Neale B, Todd-Brown K, Thomas L, Ferreira MA, Bender D, Maller J, Sklar P, de Bakker PI, Daly MJ and Sham PC: PLINK: A tool set for whole-genome association and population-based linkage analyses. Am J Hum Genet 81: 559-575, 2007.

19. DeLong ER, DeLong DM and Clarke-Pearson DL: Comparing the areas under two or more correlated receiver operating characteristic curves: A nonparametric approach. Biometrics 44: 837-845, 1988

20. Na R, Ye D, Qi J, Liu F, Lin X, Helfand BT, Brendler CB, Conran C, Gong J, Wu Y, et al: Race-specific genetic risk score is more accurate than nonrace-specific genetic risk score for predicting prostate cancer and high-grade diseases. Asian J Androl 18: 525-529, 2016.

21. Lichtenstein P, Holm NV, Verkasalo PK, Iliadou A, Kaprio J, Koskenvuo M, Pukkala E, Skytthe A and Hemminki K: Environmental and heritable factors in the causation of cancer-analyses of cohorts of twins from Sweden, Denmark, and Finland. N Engl J Med 343: 78-85, 2000.

22. Michailidou K, Hall P, Gonzalez-Neira A, Ghoussaini M, Dennis J, Milne RL, Schmidt MK, Chang-Claude J, Bojesen SE, Bolla MK, et al: Large-scale genotyping identifies 41 new loci associated with breast cancer risk. Nat Genet 45: 353-361, 2013.

This work is licensed under a Creative Commons Attribution-NonCommercial-NoDerivatives 4.0 International (CC BY-NC-ND 4.0) License. 